Sección dos: Textos

Radiografía de la innovación educativa en el Siglo XXI

\title{
La innovación educativa desde la formación permanente: Centro del profesorado de Jerez ${ }^{1}$
}

In service training providing educational innovation: Jerez Teacher Training Centre

\author{
Carmen Sotelino Polonio \\ Centro del Profesorado de Jerez \\ msotelinop@gmail.com \\ Eufemia Rosso Delgado \\ Centro del Profesorado de Jerez \\ eufemiarosso@gmail.com
}

\section{Resumen}

Este artículo pretende dar a conocer la evolución y el estado actual de la formación del profesorado no universitario en Andalucía desde la visión del Centro del Profesorado de Jerez de la Frontera (Cádiz). Se remonta a los orígenes de la formación en nuestra comunidad, hace un recorrido breve por los cambios sucesivos a lo largo de los últimos treinta años y detalla algunas de las medidas que la Consejería de Educación Andaluza ha incorporado a partir de la LEA (Ley de Educación de Andalucía 17/2007) para promover los cambios necesarios para la mejora de los centros, la calidad y el éxito educativo. Finalmente nos centramos en las actuaciones concretas que se llevan a cabo en nuestro Centro del Profesorado con el objetivo de promover la innovación en los centros educativos a través de los docentes.

Palabras clave: Formación permanente, innovación, actividad, profesorado, educación.

\begin{abstract}
This article aims to raise awareness of the evolution and current status of non-university teacher training in Andalusia from the point of view of the Jerez Teacher Training Centre. It starts with the origins of training in our community, makes a brief tour of the successive changes over the past thirty years and details some of the measures that the Andalusian Board
\end{abstract}

${ }^{1}$ Recibido: 30/01/2020 Evaluado: 11/02/2020 Aceptado: 02/04/2020 
of Education has incorporated from the LEA (Education Law in Andalucía 17/2007) to promote the changes necessary for the improvement of centres, educational quality and success. Finally, we focus on the concrete actions that are carried out in the Teachers' Centre with the aim of promoting innovation in schoolsthrough teachers.

Key words: In-service Training, innovation, training activity, teachers, education.

\section{Introducción}

Vivimos en una sociedad que está en constante cambio y los centros educativos deben adaptarse a estos continuamente para lo cual los docentes deben adquirir las competencias profesionales necesarias. Es por esto que el Estado debe ofrecer al profesorado recursos y medios que favorezcan su formación permanente. La LOE en su artículo 102 establece que "La formación permanente constituye un derecho y una obligación de todo el profesorado y una responsabilidad de las Administraciones educativas y de los propios centros”. La Junta de Andalucía en la LEA regula la formación permanente a través del Sistema Andaluz de Formación Permanente del Profesorado que se organiza en una red de Centros del Profesorado, que contarán con autonomía pedagógica y de gestión.

Este artículo pretende dar a conocer la contribución de los Centros del Profesorado a la mejora y la transformación de los centros educativos a través de la formación permanente del profesorado de los centros no universitarios de Andalucía, potenciando su desarrollo profesional a través de la adquisición de una cultura orientada a la actualización constante de su práctica docente y a la innovación educativa.

Comenzaremos explicando cómo nacen los CEP y cuál ha sido su trayectoria para que se conozca el contexto y cómo, desde su creación siempre tuvieron como objetivo la mejora y la innovación del sistema educativo. Continuaremos describiendo cuáles son las actuaciones más destacadas por parte de la Consejería de Educación y Deporte y finalizaremos detallando aquellas actuaciones que pueden destacarse como retos o apuestas por parte del CEP de Jerez.

\section{Origen y creación de los centros del profesorado}

Los Centros del Profesorado (CEP) se crearon en Andalucía en 1986, jugando un papel fundamental en la formación permanente de los docentes durante los últimos 30 años, en los que se han enfrentado a dificultades y retos, formando parte de la historia del sistema educativo andaluz y de su profesorado.

En España, durante los años setenta y principios de los ochenta florecen los Movimientos de Renovación Pedagógica (MRP) que generan un hervidero de iniciativas pedagógicas renovadoras. Destacan las Escuelas de Verano y los grupos de seguidores de las propuestas pedagógicas de Celestine Freinet (Movimiento Cooperativo de Escuelas Populares), o de Paulo Freire, los seminarios permanentes, así como el nacimiento de revistas de contenido educativo esenciales para el apoyo y el impulso a de los MRP. 
Esta prosperidad de los MRP medible por el número de personas implicadas, que llegan a cuantificarse en unos 30.000 en todo el Estado, y por la calidad de las actividades ofrecidas, sirve de base a la Administración educativa en el territorio de competencia del Ministerio de Educación y Ciencia, para tomar la decisión de crear los Centros de Profesores, en noviembre de 1984, siguiendo el modelo de los “Teachers’ centres” británicos.

En la Comunidad Autónoma Andaluza, una vez asumidas las competencias en materia educativa, se crean los CEPs cuyo funcionamiento quedó regulado por el decreto 16/1986, de 5 de febrero, definiéndolos como la institución básica destinada al perfeccionamiento del profesorado de niveles no universitarios y al fomento de programas de innovación a través del intercambio de experiencias y del encuentro y reflexión sobre el hecho educativo.

En 1992 se aprueba el I Plan Andaluz de Formación Permanente del Profesorado, basado en las necesidades de concreción del diseño curricular base en los centros y en impulsar la implantación de la LOGSE, sentando así las bases para la creación en 1997 del Sistema Andaluz de Formación, conformado por los Centros del Profesorado.

En el año 2003 se pone en marcha el II Plan de Formación como una necesidad para asegurar la calidad del aprendizaje del alumnado y el desarrollo profesional del profesorado favoreciendo la innovación y la experimentación.

Diez años más tarde, se hace indispensable continuar avanzando hacia la adaptación de la formación del profesorado a los nuevos requerimientos educativos derivados de la Ley Orgánica 2/2006, de 3 de mayo, y de la Ley 17/2007, de 10 de diciembre, así como a la consecución de los objetivos presentes en la Estrategia Europea de Educación y Formación ET2020 y se publica el Decreto 93/2013 y el III Plan Andaluz de Formación Permanente del Profesorado, como nuevo marco normativo para la formación del profesorado y como soporte para la implantación de las líneas educativas de la Consejería de Educación. Este Plan establece cinco líneas estratégicas entre las que destaca la línea III que se centra en la formación del profesorado como impulsora del conocimiento compartido y producido en los centros educativos y la investigación, la innovación educativa y las buenas prácticas.

Las grandes novedades que aporta este tercer plan son:

- La concepción del centro educativo y su profesorado como motor y elemento básico de mejora, formación e innovación, totalmente contextualizadas y con la posibilidad de su aplicación inmediata en las aulas.

- La creación de un modelo de asesorías de referencia que colaboran con los equipos directivos y los órganos de coordinación docente competentes en materia de formación de los centros.

- La consideración de la formación del profesorado como un elemento fundamental para dar respuesta a los nuevos retos educativos que plantea la sociedad actual, como factor clave para conseguir la mejora de la competencia profesional de los docentes, contribuyendo, en consecuencia, al desarrollo de una enseñanza de calidad y equidad.

- La concepción de la formación como impulsora del conocimiento compartido y producido en los centros educativos, de la investigación y la innovación educativa y 
de las buenas prácticas y como apoyo a la progresiva transformación de los centros educativos en entornos colaborativos de aprendizaje en los que participan todos los miembros de la comunidad educativa.

- La autoformación (grupos de trabajo, formaciones en centro) como la modalidad que mejor se ajusta al desarrollo de los Planes de Formación de los centros basados en el trabajo cooperativo y la investigación sobre la práctica.

- Una gran oferta formativa para el profesorado de Formación Profesional para actualizarlo y conectar la educación que se ofrezca con la realidad productiva y el empleo.

El término innovación se identifica con el de mejora, con el de cambio y transformación. En el contexto educativo Rivas Navarro (2000) define la innovación como la incorporación de algo nuevo dentro de una realidad ya existente, modificándola.

Desde esta concepción la Consejería, y en concreto la Dirección General de Formación del Profesorado e Innovación Educativa, ha tomado decisiones para asegurar la innovación en los centros educativos no universitarios, que consideramos señalar por su papel destacado en la actividad diaria de los centros:

- La convocatoria anual de medidas de apoyo al profesorado para la realización de proyectos de investigación e innovación educativa.

- La convocatoria del concurso para el fomento de la investigación y la innovación educativa, en sus dos modalidades, Joaquín Guichot y Antonio Domínguez Ortiz.

- El Portal de Buenas Prácticas Educativas.

- La puesta en marcha del programa de formación Escuelas Mentoras, como un espacio abierto de intercambio y comunicación de experiencias docentes con el que se pretende dar visibilidad a prácticas educativas innovadoras en metodología y dinámicas de aula, que servirán como referentes para otros centros.

- La convocatoria de los Planes y Programas para la Innovación Educativa para favorecer el cambio educativo, con la incorporación de metodologías activas y participativas, apostando por nuevas formas de trabajo y de organización. Surgen de la necesidad de dar respuesta a las nuevas demandas de la sociedad del siglo XXI, exigen innovación, adaptación a los cambios, flexibilidad, parten del autoconocimiento y de la experiencia de los propios individuos, generando un espacio de reflexión, fomentando el trabajo en equipo, la comunicación e impulsando a la acción para, de esta manera, estimular al alumnado y al profesorado la necesidad de formación y adaptación a las nuevas exigencias sociales. Los Planes y Programas se concretan en:

- Planes:

o Convivencia Escolar.

o Igualdad.

o Memoria Histórica y Democrática.

o Plurilingüismo.

o Profundiza. 
- Programas para la Innovación Educativa:

o Hábitos de Vida Saludable.

o Cultura Emprendedora.

o Educación Ambiental.

o Comunicación Lingüística y Bibliotecas Escolares.

o PRODIG: Programa de Digitalización de Centro.

o Programas Culturales.

Entre todos estos planes y programas merece una descripción más detallada el programa PRODIG (Programa de Digitalización de Centro) por su carácter innovador. Se pone en marcha en 2018 con el objetivo de impulsar y apoyar la transformación de los centros docentes en organizaciones educativas digitalmente competentes.

El objetivo es que los procesos de enseñanza y aprendizaje tengan lugar en un entorno tecnológico, facilitando el acceso directo a los contenidos digitales e introduciendo cambios metodológicos y organizativos que estimulen tanto al profesorado como al alumnado, permitiéndoles aprender más y mejor, gracias a la personalización de la enseñanza y a la mejora de la comunicación entre los miembros de la comunidad educativa.

\section{El centro del profesorado de Jerez y su apuesta por la innovación}

El Centro del Profesorado de Jerez forma parte de la red de Centros del Profesorado como unidades del Sistema de Formación del Profesorado no universitario de Andalucía y por tanto, sus actuaciones tienen como objetivo el desarrollo del Plan Andaluz de Formación Permanente del Profesorado incluyendo actividades que den respuesta a la concreción anual de las líneas estratégicas de la Consejería y apoyando los planes y programas de la Consejería señalados anteriormente.

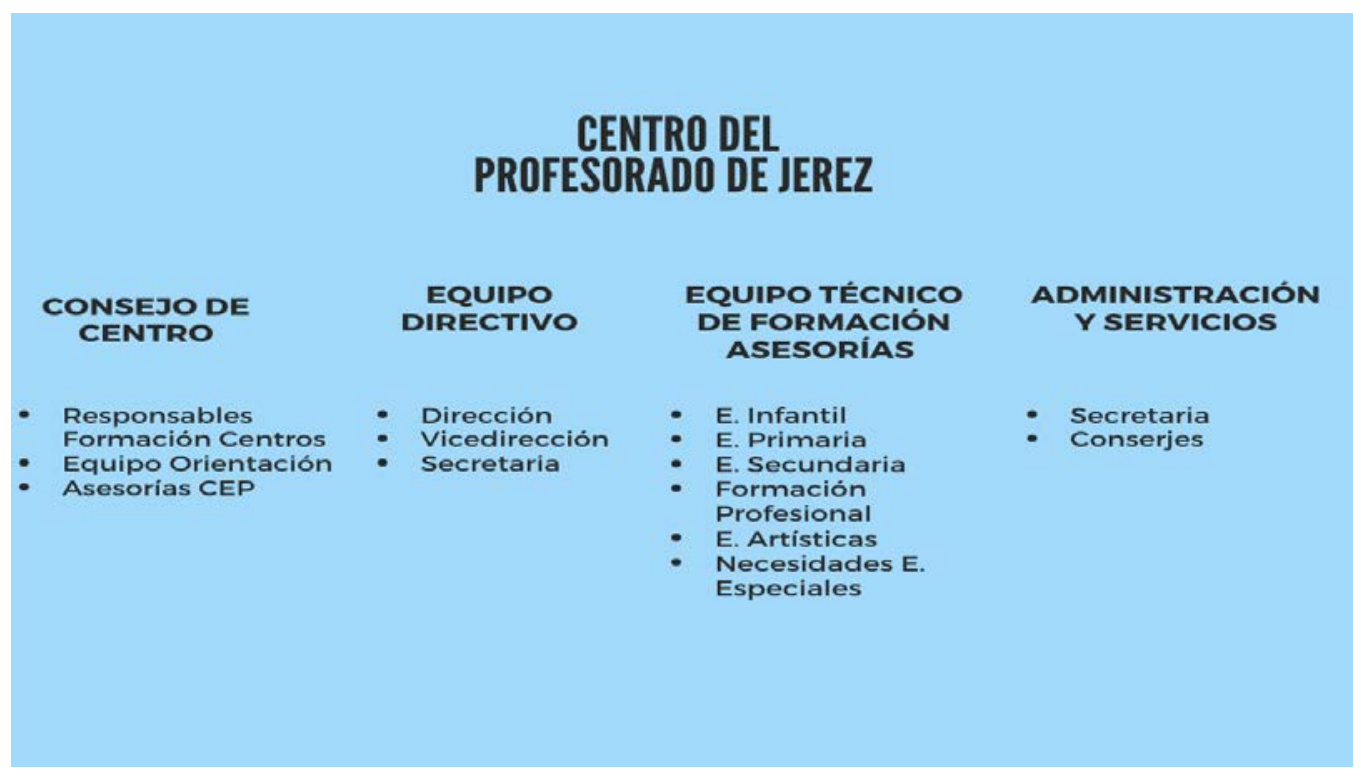

Figura 1. Organigrama CEP de Jerez 
En el organigrama anterior se observan los diferentes órganos de gobiernos y de funcionamiento del CEP.

El Consejo de Centro es el órgano de participación de los profesionales de la educación en el gobierno y gestión de los centros del profesorado. Entre otras funciones tiene la de aprobar el Plan de Centro, su gestión económica y la memoria de autoevaluación cada curso académico.

El Equipo Técnico de Formación es el órgano colegiado formado por las asesorías de formación que, en colaboración directa con el profesorado, promueve y facilita la gestión de los procesos formativos en los centros docentes.

La autonomía de pedagógica y de gestión que el Decreto 93/2013 otorga a los CEP permite al ETF trazar unas líneas de intervención propias y que son la consecuencia del proceso de detección anual de necesidades formativas de los centros que se realiza y también, de la visión y la misión que cada cuatro años se revisan y renuevan.

\section{NUESTRA VISIÓN}

Impulsar con el profesorado la mejora continua y la transformación hacia una escuela inclusiva y de calidad para favorecer el desarrollo integral del alumnado ante el reto de una sociedad en constante cambio.

\section{NUESTRA MISIÓN}

El CEP de Jerez apoya y asesora al profesorado y a los centros educativos facilitando la formación y la innovación a través de la reflexión, la investigación y el trabajo en equipo.

Estos dos elementos nos sirven de guía en la revisión y actualización anual de los objetivos que formarán, junto a las actividades formativas, el proyecto de formación. Partiendo del concepto de que el centro educativo es el motor del cambio y de la mejora, la mayoría de las actividades dan respuesta a las necesidades formativas que los centros plantean en sus planes de formación y que se vehiculan a través de las modalidades de autoformación (grupos de trabajo, formaciones en centro y formaciones específicas) que en el curso actual ascienden a 326. Las asesorías de referencia colaboran con los responsables de formación de los centros para la elaboración del plan y procuran que las actividades tengan un carácter innovador, y estén conectadas con las mejoras que los centros necesitan. Las temáticas de estas actividades están vinculadas a la introducción de metodologías activas, a la digitalización de los centros, a una evaluación acorde con el cambio metodológico, a la inclusión y a la equidad.

El Centro del Profesorado de Jerez se ha distinguido desde que se creó por su cercanía a los centros y al profesorado, por su adaptación a las necesidades de estos conforme a una sociedad en constante cambio. Una característica notable del mismo ha sido su disposición abierta hacia la innovación, por lo que nos parece que se deben destacar los retos que se han planteado en los últimos años para favorecerla: 
- La organización de actividades que promueven la innovación en los centros, a través del cambio metodológico (ABP, aprendizaje cooperativo, gamificación, flipped learning), la organización de Jornadas de Innovación en las que se presenta y se reflexiona sobre los “nuevos” espacios de aprendizaje y las últimas tendencias educativas.

- El Fomento de la creatividad y la innovación. Desde el año 2013 se impulsa desde el CEP el desarrollo de la creatividad y la innovación relacionándolas con el emprendimiento a través de la difusión de herramientas y técnicas y poniendo a disposición del profesorado recursos y materiales como el método "The Creative Platform", el uso de Lego, Visual Thinking, etc.

- La apuesta por la organización de Jornadas de Buenas Prácticas del profesorado en general, para el desarrollo de las competencias profesionales del profesorado, como reconocimiento al trabajo de los docentes y por el convencimiento de que el aprendizaje entre iguales es una estrategia de gran impacto.

- El impulso de las áreas STEAM en los centros educativos. El CEP de Jerez lleva varios años promoviendo la formación del profesorado en estas áreas, matemáticas, tecnología y ciencia. Numerosos estudios internacionales y los datos de las evaluaciones internacionales como PISA evidencian la falta de interés del alumnado de educación primaria y secundaria por las materias y carreras científicotecnológicas, especialmente entre las niñas, cuando estas áreas son y serán fundamentales en la sociedad del siglo XXI. La acciones del CEP se materializan en: la organización de la Feria de la Ciencia en la Calle que es un gran evento que moviliza a miles de alumnos y alumnas cada curso y a centenares de docentes y que permite a los centros educativos mostrar la investigación y las experiencias científicas y tecnológicas que realizan con el alumnado, las abre a la sociedad y las pone en contacto con el mundo académico universitario y con las empresas; la formación en robótica y pensamiento computacional; la formación del profesorado para el diseño de proyectos de investigación científica en el aula, técnicas de laboratorio, proyectos medioambientales, nuevas perspectivas metodológicas en la enseñanza de las matemáticas, etc.

- El fomento del Flamenco en la Escuela, con la organización de unas Jornadas provinciales desde el año 2002, cursos de flamenco y apoyo a las iniciativas de los centros y grupos de docentes interesados en estas temáticas.

- La promoción de proyectos artísticos en los centros educativos, partiendo de que, como señala María Acaso, “la educación artística es un motor de cambio social”. Esta apuesta se concreta con actividades de teatro, apoyo a la Escuela de Arte y a los conservatorios, y actividades que tienen el objetivo de sensibilizar a los centros para que pongan en valor la importancia del arte para el desarrollo integral del alumnado.

- El apoyo decidido al profesorado dotándolo de estrategias para la atención a la diversidad creciente en las aulas. Llevamos varios años en los que, además de atender al profesorado de alumnado con necesidades especiales de apoyo educativo, se organizan actividades para el profesorado de aulas ordinarias presentando el enfoque denominado Diseño Universal para el Aprendizaje (DUA), desarrollado por el Center for Applied Special Technology (Centro de Tecnología Especial Aplicada, CAST).

- La internacionalización que se concreta en la organización y participación en eventos y proyectos europeos y en el asesoramiento a los centros de la zona para facilitar su 
implicación en los mismos, así como en el apoyo al Plan de Plurilingüismo a través de numerosos grupos de trabajo y cursos sobre actualización lingüística, formación sobre metodología AICLE y jornadas de buenas prácticas para el profesorado de lenguas extranjeras y el de áreas no lingüísticas de centros plurilingües.

- Medidas que aseguren una educación basada en la equidad: igualdad y diversidad de género, el uso de un lenguaje inclusivo e igualitario.

Podríamos mencionar igualmente las actuaciones que se desarrollan para apoyar la digitalización de los centros formando a los docentes en el uso de recursos digitales para posibilitar el uso de metodologías activas en las aulas y de una evaluación acorde con el trabajo por competencias con el alumnado, para la gestión y organización de los centros y la comunicación con las familias, etc.

\section{Datos del proyecto de formación del CEP de Jerez, en el curso 2018/2019}

Modalidades Formativas:

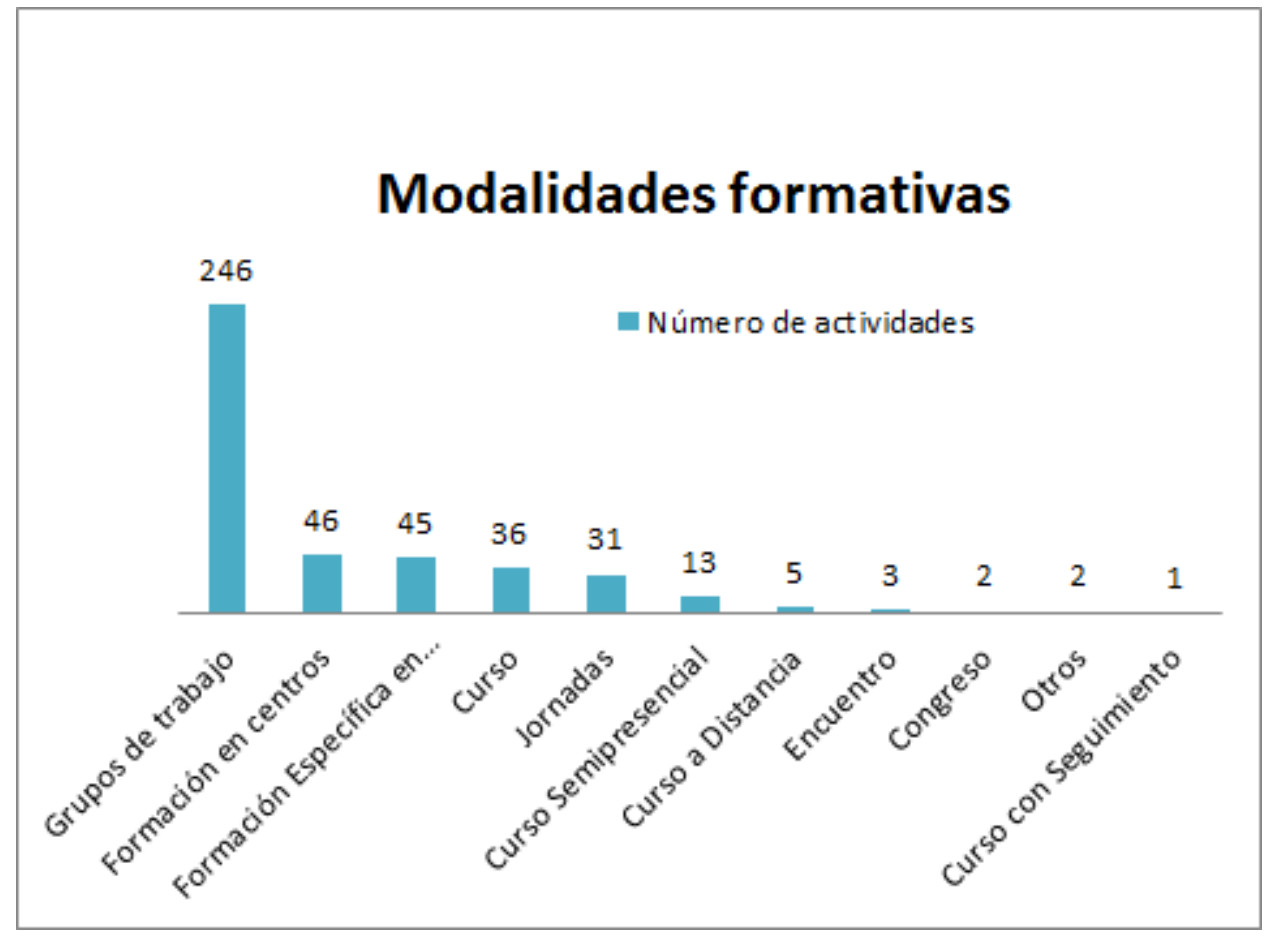

Figura 2. Modalidades formativas

Como se observa en el gráfico prevalecen las actividades de autoformación (grupos de trabajo, formación en centro y formaciones específicas) que parten de la iniciativa de los docentes de un mismo centro para conseguir sus objetivos de mejora. En cuanto a la formación a distancia se debe señalar que la Consejería oferta anualmente alrededor de ochenta cursos en dos convocatorias de esta modalidad a través del aula Virtual de Formación del Profesorado, además de los 84 cursos que permanecen en abierto en la misma, con temáticas diversas que se agrupan en las siguientes temáticas: 
"Competencias Clave; Plurilingüismo;TIC; Escuela Inclusiva; Desarrollo profesional (Competencias docentes, Competencias específicas, Función orientadora, Didáctica y gestión aula), Participación de la comunidad; Investigación e innovación”.

Número de actividades y asistentes:

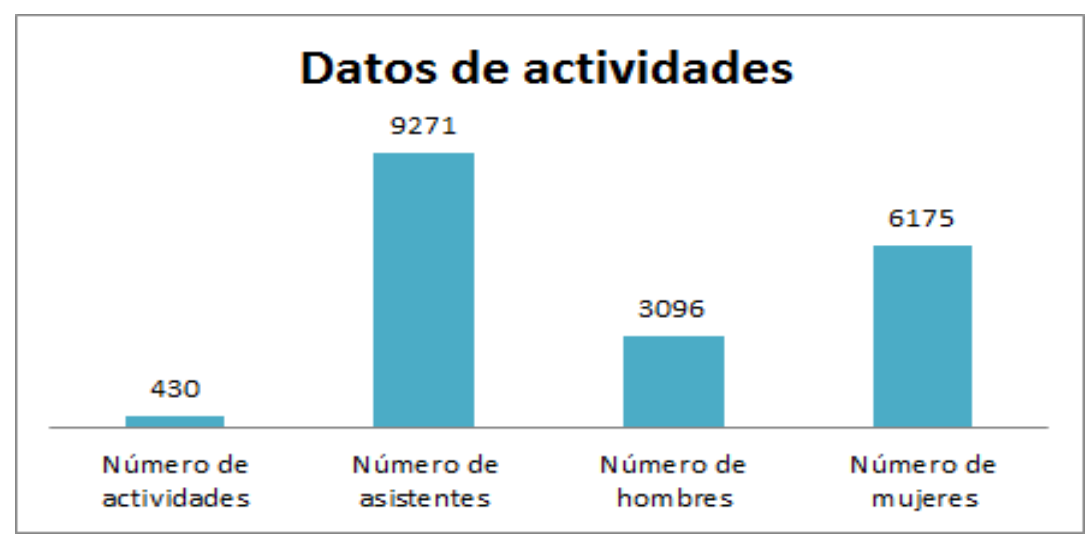

En este gráfico se recogen el número total de actividades organizadas durante el curso 2018/2019 que asciende a 430 de cualquier modalidad. En cuanto al número de asistentes a las actividades destaca que el porcentaje de mujeres duplica al de hombres.

Temáticas de las actividades formativas:

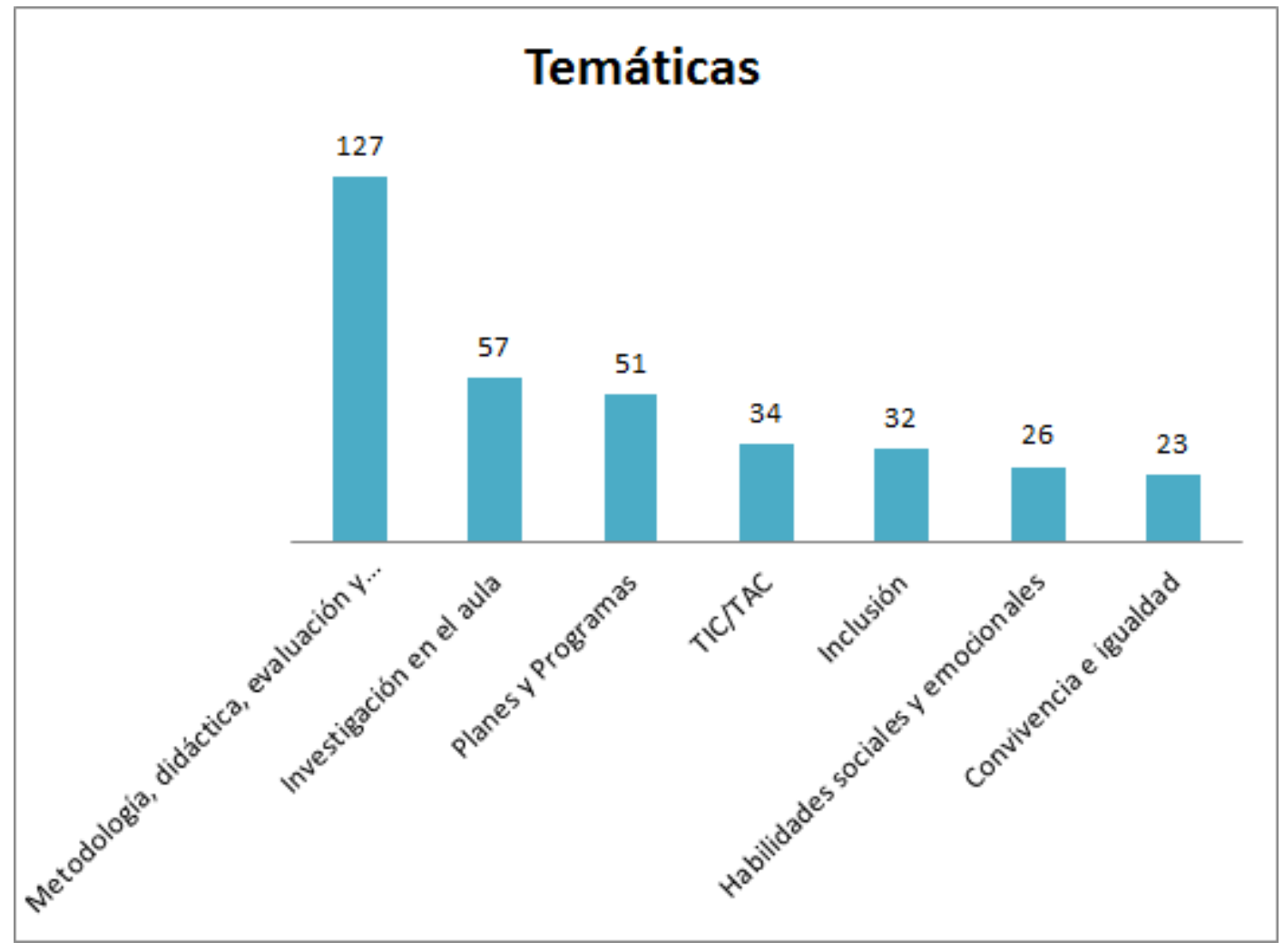


Como se observa en el gráfico se organizan un gran número de actividades relacionadas con la metodología, la evaluación y las competencias clave del alumnado porque es una demanda del profesorado y una necesidad básica para la transformación de los centros; las actividades de investigación en el aula están mayoritariamente vinculadas a la organización de la Feria de la Ciencia en la Calle; también se organizan actividades para el desarrollo de los Planes y Programas de Innovación Educativa.

\section{Conclusiones}

Concluimos recordando que el profesorado constituye la pieza clave del cambio y de la innovación educativa por lo que su formación debe ser de alta calidad, y ese es el papel de los CEP que tenemos la responsabilidad de fomentar la capacidad de innovación, reflexión y crítica en los docentes para que tengan recursos que puedan dar respuesta a una realidad educativa cambiante y, a veces, complicada, con exigencias y demandas que necesitan de una actualización constante

Esperamos con este artículo haber sido capaces de transmitir el esfuerzo y el dinamismo existente en la formación del profesorado de nuestra zona y que puede hacerse extensible al resto de los Centros del Profesorado de Andalucía, a partir de las líneas estratégicas de la Consejería de Educación y Deporte y de las necesidades de los centros y de los docentes de nuestra comunidad autónoma.

\section{Referencias}

Acaso, M. \& Megías, C. (2017). Art Thinking. Barcelona. Paidós Educación.

Carbonell, J. (2015). Pedagogías del siglo XXI. Alternativas para la innovación educativa ( $3^{a}$ Edición). Barcelona: Octaedro Editorial.

Díaz Negrín, M. (2014). La formación Permanente del Profesorado. Análisis y Sentido. EL GUINIGUADA, Revista de investigaciones y experiencias en Ciencias de la Educación. N ${ }^{\circ}$ 23, 53-62.

Hernández Díaz, J.M. (2011). La Renovación pedagógica en España al final de la transición. El encuentro de los movimientos de renovación pedagógica y el ministro Maravall. Educació i Historia:Revista d’ Història de l’Educació, 18, 81-105.

Iglesias Martínez, M. J., Lozano Cabezas, I. y Roldán Soler, I. (2018). La calidad e innovación educativa en la formación continua docente: un estudio cualitativo en dos centros educativos. Organización de Estados Iberoamericanos para la Educación, la Ciencia y la Cultura (OEI-CAEU).

Imbernón, F. (2007). 10 ideas clave: la formación permanente del profesorado. Barcelona: GRAO. 
Marcelo García, C., Mayor Ruiz, C., Gallego Noche, B. (2010). Innovación educativa en España desde el punto de vista de sus protagonistas. Revista de currículum y formación del profesorado. Vo.14, N. 1, pp. 111-134.

Montero, L. (2006). Las instituciones de formación permanente, los formadores y las políticas de formación en el Estado de las Autonomías. En Escudero, J. y Alberto, L (Coords.) La formación del profesorado y la mejora de la educación. Barcelona: Octaedro.

Muñoz. J.M.E. (2017). La formación continua del profesorado de la educación obligatoria en el contexto español. Revista de Currículum y Formación de Profesorado. Vol. 21, núm. 3, pp. 1-20

Rivas Navarro, M. (2000). Innovación educativa: teoría, procesos y estrategias. Madrid:Síntesis

Legislación Educativa

La Ley Orgánica de Educación (LOE) 2/2006, de 3 de mayo. BOE.

LEY 17/2007, de 10 de diciembre, de Educación de Andalucía. BOJA.

Orden de 31 de julio de 2014, por la que se aprueba el III Plan Andaluz de Formación Permanente del Profesorado. BOJA.

Decreto 93/2013, de 27 de agosto, por el que se regula la formación inicial y permanente del profesorado en la Comunidad Autónoma de Andalucía, así como el Sistema Andaluz de Formación Permanente del Profesorado. BOJA.

Páginas Web de Referencia:

Buenas prácticas educativas (CEP Jerez): http://bit.ly/2O0qYYd

Planes y programas educativos:http://bit.ly/3121q2c

CEP de Jerez: http://bit.ly/2Objd1w

Feria de la Ciencia en la Calle: http://bit.ly/2RBOTiG

Aula Virtual de Formación del Profesorado: http://bit.ly/32Q2jfe 